\title{
Parameter Optimization Method for the Pastor-Zienkiewicz Model Based on Empirical Mode Decomposition and Cloud Theory
}

\author{
Zhang Hongyang ${ }^{1,2}$, Zhang Wenxin ${ }^{1}$, Ding Zelin ${ }^{1, *}$, Li Shuai $^{1}$ and Ge Wei ${ }^{3}$ \\ ${ }^{1}$ College of Water Conservancy, North China University of Water Resources and Electric Power, Zhengzhou 450046, China \\ ${ }^{2}$ Collaborative Innovation Center of Water Resources Efficient Utilization and Protection Engineering of Henan Province, Zhengzhou \\ 450046, China \\ ${ }^{3}$ Faculty of Technology, Policy and Management, Delft University of Technology, Delft 2628 BX, the Netherlands
}

Received 28 May 2019; Accepted 21 December 2019

\begin{abstract}
The key to simulating the mechanical properties of earth-rockfill dam materials is selecting a reasonable constitutive model. The traditional constitutive model targets specific earth-rockfill dam materials and involves many hypotheses in theory, resulting in large common errors in applications. The Pastor-Zienkiewicz model (P-Z model) based on generalized plastic mechanics theory has a strict theoretical base, but it involves many parameters. Moreover, the determination of parameter $\alpha$ entails high discreteness because of experimental conditions, and this discreteness influences the accuracy of simulation analysis. The mechanical properties of earth-rockfill dam materials were simulated using the P-Z model in this study to optimize parameter $\alpha$ of the model and decrease its discreteness. First, conventional triaxial shear test results of earth-rock materials were denoised with the empirical mode decomposition method. Second, a method to optimize parameter $\alpha$ was proposed by using cloud theory. Lastly, a numerical simulation analysis of the triaxial shearing test was performed with the optimized P-Z model. Research results demonstrate that the entropy error and hyper entropy error rates of the triaxial shearing test data decline by $35 \%$ and $67 \%$, respectively, and the cloud droplet range of parameter $\alpha$ is narrowed by $32 \%$. The discreteness of the values decreases significantly. The simulation calculation results are consistent with the test results, thereby confirming the reasonability of the proposed parameter optimization method. The optimized P-Z model can simulate the mechanical properties of earth-rockfill dam materials accurately. This study can provide a theoretical reference for finite element simulation analysis of earth-rockfill dam structures.
\end{abstract}

Keywords: Pastor-Zienkiewicz model, Parameter $\alpha$, Empirical mode decomposition EMD, Cloud theory, parameters optimization

\section{Introduction}

With the implementation of the development of the western region in china and West-East electricity transmission project, many high earth-rockfill dams are expected to be constructed in Southwest China, including Yunnan, Guizhou, and Sichuan. The major earth-rockfill dams include the corewall rockfill dam in Changhe Hydropower Station (height of $240 \mathrm{~m}$ ), the soil core-wall rockfill dam in Lianghekou Hydropower Station (height of $295 \mathrm{~m}$ ), and the soil corewall rockfill dam in Shuangjiangkou Hydropower Station (height of $314 \mathrm{~m}$ ) [1]. The mechanical properties of earthrockfill dam materials change continuously in response to complicated natural conditions and various external forces, leading to different degrees of aging, dam lesion and cracks, and even catastrophic failures (e.g., dam outburst) as time passes. Therefore, studying the mechanical properties of earth-rockfill dam materials and protecting the operational safety of dams are significant.

The constitutive model is an important factor that influences the analysis results of the mechanical properties of earthrockfill dam materials. Traditional constitutive models can be divided into two types. The first type encompasses

*E-mail address: dingzelin@126.com

ISSN: $1791-2377$ @ 2019 School of Science, HU. All rights reserved.

doi:10.25103/jestr.126.06 nonlinear elastic models, including Duncan-Chang [2] and Naylork-G models [3]. The second type involves elasticplastic models and primarily includes Drucker-Prager [4], Cambridge [5], and Shenzhujiang double-yield surface models [6]. A nonlinear elastic model can reflect the main change laws of soil mass, but it cannot reflect the dilatancy of soils and the effects of the stress path and intermediate principal stress on deformation. An elastic-plastic model divides the deformation of materials into elastic and plastic deformation. It calculates elastic deformation via Hooke's law and plastic deformation via plastic theory. This model can reflect the complicated anisotropism, dilatancy, and other mechanical properties of soil mass, but it assumes that the incremental direction of plastic stress is only related to stress and not to stress increment. The associated flow rule is applied, and the principal stress axis rotation is ignored, which differs significantly from practical situations. Therefore, traditional constitutive models cannot simulate the mechanical properties of earth-rockfill dam materials accurately.

With the development of test and computer technologies for earth-rockfill dam materials, Pastor and Zienkiewicz constructed a constitutive model called Pastor-Zienkiewicz model (P-Z model) based on the theory of generalized plastic mechanics [7] in 1985. The P-Z model has explicit physical significance and can describe the static and 
dynamic mechanical properties of earth-rockfill dam materials. Moreover, in this model, yield and plastic potential surfaces do not need to be defined for plastic deformation to be deduced. Instead, the model determines yield and plastic potential surfaces via the vectors of loading and loading-unloading directions of plastic potentials, thereby increasing the convenience of simulating the mechanical properties of earth-rockfill dam materials. However, the P-Z model involves many material parameters. Parameter $\alpha$, which represents Poisson's ratio in the P-Z model, often entails large discreteness during parameter deduction based on conventional triaxial shear test results. This large discreteness further affects the accuracy of the P$\mathrm{Z}$ model in simulating the mechanical properties of earthrockfill dam materials.

Therefore, in the current study, parameter $\alpha$ of the P-Z model was optimized with the empirical mode decomposition (EMD) method and cloud theory on the basis of the triaxial shear test results of earth-rockfill dam materials to decrease the model's discreteness. This optimization could improve the accuracy of the P-Z model in simulating the mechanical properties of earth-rockfill dam materials.

\section{State of the art}

Studying the mechanical properties of earth-rockfill dam materials by using the generalized plastic mechanical model is still in the preliminary stage in the 20th century due to the complexity of these materials. In 1984, Zienkiewicz and Morz performed a test on clay [8,9] and built a generalized plastic mechanical theoretical system. They separately defined the yield and plastic potential surfaces of materials under stress and explained their relations. The yield and plastic potential surfaces were described with the vectors of unloading direction and plastic potential direction. In 1985, Pastor and Zienkiewicz constructed the P-Z model on the basis of generalized plastic mechanical theory. Subsequently, Morz et al. [10] conducted a series of studies based on the P$\mathrm{Z}$ model. However, the $\mathrm{P}-\mathrm{Z}$ model cannot easily determine the effects of key mechanical properties, such as compactness and confining pressure, on cohesionless soil. Pastor [11] introduced parameter $\alpha$, which involves Poisson's ratio, to describe the critical state theory of the P-Z model. The value of parameter $\alpha$ can influence the positions of plastic potential and yield surfaces and their relations. Consequently, the accuracy and reliability of mechanical properties simulated by the P-Z model are affected. However, determining parameter $\alpha$ involves considerable randomness and mutability. Jefferies [12] et al. modified relevant parameters in the P-Z model and achieved good simulation results, but they failed to determine and verify the main factor that influences $\alpha$. With the development of test and computer technologies in the $21 \mathrm{st}$ century, studies on the mechanical properties of earthrockfill dam materials by using the $\mathrm{P}-\mathrm{Z}$ model have entered a new stage. Zhang Hong Yang $[13,14]$ deduced the static and dynamic parameters of P-Z models according to the results of a triaxial test on clay and sandy soils and a vibrostand test. The obtained static and dynamic parameters were applied in the numerical analysis of practical engineering to discuss the safety of earth-rockfill dams under static and dynamic conditions. However, the mean values of the model parameters were determined in the presence of discreteness, and the test data involved considerable noise with a certain randomness. Using the P-Z model, Chen Shengshui [15] analyzed the deformation features of rockfill materials under constant and varying amplitudes of stress cyclic loading processes. Although the effects of cumulative volumetric strain on dilatancy were considered, the model parameters in the calculation were still determined through conventional indoor monotonous and cyclic loading tests. The researchers failed to provide a solution to the verification of parameter discreteness. In summary, research on the factors that influence the accuracy of the P-Z model focused on noise influence of triaxial test data and discreteness of parameters. The mechanical properties of earth-rockfill dam materials can be simulated effectively only when the problems in these aspects are solved.

The EMD method is a common data denoising technique [16]. Its working principle is as follows: a time series is decomposed into several intrinsic mode functions (IMFs), and subsequences obtained from the IMFs are used to denoise unsteady data, thereby acquiring a series of relatively stable time sequences. Zhang, Jianwei and Bao, Zhenlei et al. [17] applied the EMD method to vibration denoising caused by the leakage of flood-releasing structures. Certain amounts of noise were eliminated with the singular value decomposition method, and IMF was calculated with the EMD method. As a result, the effects of irregular noises on the vibration of flood-releasing structures were eliminated effectively. Li Chengye and Lian Jijian [18] conducted a steady analysis of measured vibration data by using EMD and wavelet threshold methods. They determined the model parameters of flood-releasing structures via natural excitation technique and HilbertHuang transform methods after denoising and verified these methods through a case study of a practical project, namely, Ertan Arc Dam. Good vibration coupling results were obtained. Su Huaizhi and Yang Meng et al. [19] decomposed the deformation parameters of an earth-rockfill dam slope by using the EMD method and extracted alarming indexes of accelerating slope deformation. They compared the results with measurement data and observed significant denoising effects. These studies have shown that data denoising with the EMD method can eliminate the interference factors of data and enhance the data trend. The EMD method was used resolve data noises in the triaxial shear test in the present study.

Cloud theory was proposed by Li Deyi in 1996 [20]. This theory can provide a quantitative description of uncertain indexes $[21,22,23]$ and has become the main approach to solve the uncertainty problems of multiple factors. Many scholars have conducted relevant studies on the uncertainty of dams on the basis of cloud theory. Tan Zhiying [24] calculated the total weights of the safety evaluation index of a dam by using the cloud theory model and game theory, which improved the accuracy of model assessment and the extensiveness of the application. Zhang Tao [25] predicted dam deformation by using the cloud theory model and compared it with engineering measurement. High consistency was noted. With consideration of uncertain influences of many factors on the compaction quality of dams during the construction, Wang $\mathrm{F}$ and Zhong DH et al. [26] constructed a new compaction evaluation system by combining the cloud theory model and fuzzy neural network. The results of this system were compared with field sampling results, and high consistency with engineering practices was obtained. In summary, the data analysis method based on cloud theory can predict the 


\section{Zhang Hongyang, Zhang Wenxin, Ding Zelin, Li Shuai and Ge Wei/ \\ Journal of Engineering Science and Technology Review 12 (6) (2019) 44 - 53}

uncertainty of research objects under the effects of multiple factors, and it can be applied to solve the discreteness of $\mathrm{P}-\mathrm{Z}$ model parameters, which are sensitive to numerous factors.

To address the shortcomings of existing studies, this study optimized the main parameter $\alpha$, which can influence the accuracy of the $\mathrm{P}-\mathrm{Z}$ model when it is used to simulate the mechanical properties of earth-rockfill dam materials based on the EMD method and cloud theory. In other words, on the basis of the triaxial shear test data of coarse particles, this study denoised the test data by using the EMD method and processed and optimized the calculation formula of parameter $\alpha$ of the P-Z model by referring to cloud theory to reduce the discreteness. The increase in the accuracy of the P-Z model lays the foundation for the accurate simulation of the mechanical properties of earth-rockfill dam materials.

The remainder of this study is organized as follows. Section 3 introduces the basic principles of the P-Z model, EMD method, and cloud theory and elaborates the optimization method for the calculation formula of parameter $\alpha$. Section 4 presents the formula of parameter $\alpha$ and a simulation analysis of triaxial test data. Section 5 summarizes the conclusions.

\section{Methodology}

\subsection{General framework}

In the P-Z model, it is assumed that deformation of the material can be considered as the result of deformations produced by $\mathrm{M}$ separate mechanisms, all of these subjected to the same state of stress (series models).

The strain increment can thus be written as:

$$
d \varepsilon=\sum_{m=1}^{M} d \varepsilon^{(m)}
$$

where

$$
d \varepsilon=\sum_{m=1}^{M} C^{(m)}: d \sigma
$$

and

$$
C=\sum_{m=1}^{M} C^{(m)}
$$

The total increment of strain is composed of the increment of $M$ separate mechanisms:

$$
\begin{aligned}
& d \varepsilon \\
& =\sum_{m=1}^{M} C^{e(m)}: d \sigma+\sum_{m=1}^{M} \frac{1}{H_{L / U}^{(m)}}\left[n_{g L / U}^{(m)} \otimes n^{(m)}\right]: d \sigma
\end{aligned}
$$

or

$$
\begin{aligned}
& d \varepsilon=C^{e}: d \sigma+\sum_{m=1}^{M} \frac{1}{H_{L / U}^{(m)}}\left[n_{g L / U}^{(m)} \otimes n^{(m)}\right]: d \sigma \\
& d \varepsilon=C_{L / U}^{e / p}: d \sigma
\end{aligned}
$$

where $C^{e / p}$ is the inverse matrix of the stiffness matrix $D^{e / p}$.

\subsubsection{Stress and strain invariants}

In the P-Z model, the effective stress is expressed in the form of stress tensor as:

$$
\begin{aligned}
& I_{1}=\frac{1}{3} \sigma_{i i}^{\prime} \\
& J_{2}=\frac{1}{2} S_{i j} S_{j i} \\
& J_{3}=\frac{1}{3} S_{i j} S_{j k} S_{k i}
\end{aligned}
$$

where $S_{i j}=\sigma^{\prime}{ }_{i j}-\delta_{i j} I_{1}, I_{1}$ is the first stress invariants, $J_{2}$ is the second invariant of the stress deviator, and $J_{3}$ is the third invariant of the stress deviator According to the basic principle of the finite element method:

$$
\begin{aligned}
& p=-I_{1} \\
& q=\sqrt{3 J_{2}} \\
& \theta=-\frac{1}{3} \sin ^{-1}\left(\frac{3 \sqrt{3}}{2} \frac{J_{3}}{J_{2}^{3 / 2}}\right)
\end{aligned}
$$

where $\theta$ is the Lode's angle, $\theta \in\left(-\frac{\pi}{6}, \frac{\pi}{6}\right), p$ is the generalized principal stress, and $q$ is the generalized shear stress. Corresponding to the stress invariant, the strain invariant is expressed as:

$$
\begin{aligned}
& d \varepsilon_{v}=d \varepsilon_{i i} \\
& d \varepsilon_{S}=\frac{2}{3}\left(\frac{1}{2} d e_{i j} d e_{j i}\right)^{1 / 2}
\end{aligned}
$$

where $d e_{i j}=d \varepsilon_{i j}-\frac{1}{3} \delta_{i j} d \varepsilon_{k k}, \varepsilon_{v}$ is the volumetric strain, $\varepsilon_{s}$ is the shear strain, and $\delta_{i j}$ is the Kronecker index.

$$
\delta=E=\left[\begin{array}{ccc}
1 & \ldots & 0 \\
\ldots & 1 & \ldots \\
0 & \ldots & 1
\end{array}\right]
$$

In the triaxial test, there are:

$$
\begin{gathered}
p=\frac{1}{3}\left(\sigma_{1}+2 \sigma_{3}\right), q=\sqrt{3 J_{2}}=-\left(\sigma_{1}-\sigma_{3}\right) \\
\varepsilon_{v}=\varepsilon_{1}+2 \varepsilon_{3}, \varepsilon_{s}=\frac{2}{3}\left(\varepsilon_{1}-\varepsilon_{3}\right)
\end{gathered}
$$

where $\sigma_{1}$ is the maximum principal stress, $\sigma_{3}$ is the minimum principal stress, and $\left(\sigma_{1}-\sigma_{3}\right)$ is the stress deviator.

\subsubsection{Loading plastic potential direction vector}

Assuming that the elastic strain is far smaller than the plastic strain, the expansion coefficient of soil $d$ is: 
$d=\frac{d \varepsilon_{v}^{p}}{d \varepsilon_{s}^{p}} \approx \frac{d \varepsilon_{v}}{d \varepsilon_{s}}$

Pastor proposed a new formula based on the critical state theory, expressing the ratio of soil expansion ratio to stress $\eta(\eta=q / p)$ as:

$d=(1+\alpha)(M-\eta)$

In order to distinguish the plastic potential direction vector from the loading direction vector, in this study, the formula (12) is written as:

$d_{g}=\left(1+\alpha_{g}\right)\left(M_{g}-\eta\right)$

where $\alpha_{g}$ is the material parameter, $M_{g}$ is the slope of the critical state line.

In the $(p, q)$ two-dimensional space, loading plastic potential direction vector is $n_{g_{+}}=\left(n_{g_{+}}^{p}, n_{g_{+}}^{q}\right)^{T}$ as:

$$
\begin{aligned}
& n_{g+}^{p}=d_{g}=\left(1+\alpha_{g}\right)\left(M_{g}-\eta\right) \\
& n_{g-}^{p}=1
\end{aligned}
$$

According to the optimization algorithm proposed by $\mathrm{Li}$ Hongen, it can be expressed as:

$$
\begin{aligned}
& n_{g+}^{p}=\frac{d_{g}}{\sqrt{1+d_{g}^{2}}} \\
& n_{g-}^{p}=\frac{1}{\sqrt{1+d_{g}^{2}}}
\end{aligned}
$$

As can be seen from the formulas (14) and (15), the plastic potential direction is not determined by the plastic potential surface. Integrating the formula (15) to obtain:

$$
G=q-M_{g} p\left(1+\frac{1}{\alpha_{g}}\right)\left[1-\left(\frac{p}{p_{g}}\right)^{\alpha_{g}}\right]=0
$$

where $p_{g}$ is an integral constant. With the above foundation,the $(p, q)$ two-dimensional space can be expanded into $(p, q, \theta)$ three-dimensional space to get $n_{g_{+}}=\left(n_{g_{+}}^{p}, n_{g+}^{q}, n_{g+}^{\theta}\right)^{T}$ as:

$$
\begin{aligned}
& n_{g+}^{p}=\left(1+\alpha_{g}\right)\left(M_{g}-\eta\right) \\
& n_{g+}^{q}=1 \\
& n_{g+}^{\theta}=\frac{\partial G}{\partial \theta}=-\frac{1}{2} q M_{g} \cos 3 \theta
\end{aligned}
$$

The unloading plastic potential direction vector $n_{g_{-}}$is expressed as $n_{g_{-}}=\left(n_{g_{-}}^{p}, n_{g_{-}}^{q}, n_{g_{-}}^{\theta}\right)^{T}$ in the $(p, q, \theta)$ three- dimensional space,which is opposite to the loading plastic potential direction vector only in the $\mathrm{p}$ direction, and the same in the other two directions, as shown in the following formula:

$$
\begin{aligned}
& n_{g-}^{p}=-\left|n_{g+}^{p}\right| n_{g+}^{p}=-\left|\left(1+\alpha_{g}\right)\left(M_{g}-\eta\right)\right| \\
& n_{g-}^{q}=n_{g+}^{q}=1 \\
& n_{g-}^{\theta}=n_{g+}^{\theta}=-\frac{1}{2} q M_{g} \cos 3 \theta
\end{aligned}
$$

\subsubsection{Loading direction vector}

$n$ is used in this study to represent the loading direction vector to distinguish the plastic potential direction vector $n_{g+/-}$, so there is $n=\left(n^{p}, n^{q}, n^{\theta}\right)^{T}$ in the $(p, q, \theta)$ threedimensional space, similar to the plastic potential direction vector $n_{g+/-}$. The loading direction vector can be expressed as:

$$
\begin{aligned}
& n^{p}=\left(1+\alpha_{f}\right)\left(M_{f}-\eta\right) \\
& n^{q}=1 \\
& n^{\theta}=-\frac{1}{2} q M_{f} \cos 3 \theta
\end{aligned}
$$

$\alpha_{f}$ and $M_{f}$ in formula (19) are different as $\alpha_{g}$ and $M_{g}$ in formula (17). The P-Z model applies non-associative flow rule. It can be concluded from formula (20) that the loading direction vector $\mathrm{n}$ is not built on the yield surface. Similarly, the integration of the loading direction vector ${ }_{n}$ results in:

$$
F=q-M_{f} p\left(1+\frac{1}{\alpha_{f}}\right)\left[1-\left(\frac{p}{p_{f}}\right)^{\alpha_{f}}\right]=0
$$

where $P_{f}$ is an integral constant.

\subsection{EMD method}

The basic principle of the EMD method is as follows [27]:

(1) For time sequence $F(t)$, its maximum ( $\left.t_{\max }\right)$ and minimum $\left(t_{\min }\right)$ are obtained and fitted by a spline (SPL) to form the upper and lower envelopes. The mean function $(m(t))$ of the upper and lower envelopes is calculated. $\varphi(t)$ is computed according to the formula $\varphi(t)=F(t)-m(t)$. If $\varphi(t)$ satisfies two features of IMF simultaneously, then it represents one IMF component of $F(t)$ and is set to $I_{1}(t)$. If $\varphi(t)$ cannot satisfy two features of IMF simultaneously, then $\varphi(t)$ can be viewed as the original time sequence function, and the above calculation is repeated until a satisfactory $I_{1}(t)$ is obtained;

(2) IMF functions $I_{1}(t)$ are separated from the original time sequence $r(t)=F(t)-I_{1}(t)$, thus obtaining the residual function $r(t)$;

(3) The residual function $r(t)$ is used as a new $F(t)$ to repeat the steps above, thereby obtaining the second 
component $I_{2}(t)$ that satisfies two features of IMF. The above steps are repeated until IMF components can no longer be extracted from $r(t)$. Finally, several IMFs and one residual function formula are obtained as:

$$
F(t)=\sum_{i=1}^{n} I_{i}(t)+r(t)
$$

\subsection{Basic principle of cloud theory}

Cloud theory is the specific implementation method of cloud, and it represents an integral concept with three digital features: expectation ( $E x)$, entropy ( $E n)$, and hyper entropy ( $\mathrm{He}$ ) [28,29]. Ex is the measurement of the basic certainty of the qualitative concept, and it presents the most typical sample of the concept. En is determined by the randomness and fuzziness of the concept. It reflects the degree of uncertainty of the expected value and represents the discreteness degree and value range of cloud droplets. He measures the uncertainty of entropy. It is the entropy of entropy and represents the discreteness degree of entropy. Cloud theory covers forward and reverse cloud algorithms. Cloud algorithms are realized through a cloud generator. A forward cloud algorithm realizes transformation from a qualitative concept to a quantitative expression, whereas a reverse cloud algorithm realizes transformation from a quantitative expression to a qualitative concept. Cloud theory is applied for mutual transformation between a qualitative concept and a quantitative expression, thus forming mutual mapping between the two.

Cloud theory defines an uncertain sequence as sequence $U$, and $C$ is the qualitative judgment of $U$ [30,31]. If a magnitude is given, $x \in U$ and $x$ is a random implementation of $C$, then the certainty $(\mu(x) \in[0,1])$ of $x$ about $C$ is a random number with steady bias. If $\mu: U \rightarrow[0,1](\forall x \in U, x \rightarrow \mu(x))$, then the distribution of $x$ on $U$ forms the cloud, and $x$ becomes the cloud droplet.

Cloud theory reflects the overall characteristics by introducing $\mathrm{Ex}, \mathrm{En}$, and $\mathrm{He}$. Eigenvalue statistics on monitoring effect size were obtained in this study through a reverse cloud generator. The principle of the reverse cloud generator is:

(1) Calculate the sample mean $\bar{X}=\frac{1}{n} \sum_{i=1}^{n} x_{i}$ and variance $S^{2}=\frac{1}{n-1} \sum_{i=1}^{n}\left(x_{i}-\bar{X}\right)$ from $x_{i}$

(2) Expected value $E x=\bar{X}$, entropy $E n=\sqrt{\frac{\pi}{2}} \times \frac{1}{n} \sum_{i=1}^{n}\left|x_{i}-E x\right|$ and $\mu=e^{-\frac{(x-E x)^{2}}{2(E n)^{2}}}$

(3) Get hyper entropy $H e=\sqrt{S^{2}-E n^{2}}$.

Forward cloud generator can realize the quantitative conversion of the qualitative concept, which generates cloud drop groups based on the expectation, entropy, and hyper entropy of $x_{i}$. The specific principle is:

(1) Generate an automatic random number $E n^{\prime}$ with $E x$ as the expectation and $H e^{2}$ as the variance according to the digital feature $(\mathrm{Ex}, \mathrm{En}, \mathrm{He})$;

(2) Taking $E x$ as the expectation and $E n^{\prime}$ as the variance, generate a normal random number $x$, which is called a cloud drop on $U$;
(3) Calculate $\mu=e^{-\frac{(x-E x)^{2}}{2(E n)^{2}}}$, and $\mu_{i}$ is the degree of certainty that $x_{i}$ belongs to $C$;

(4) Repeat the above steps until $n$ cloud drops are generated.

\subsection{Formula optimization of Parameter}

The derivation formula of $\alpha$ is obtained according to formula (13):

$$
\alpha_{g}=\frac{d \varepsilon_{v}}{d \varepsilon_{s}}\left(M-\frac{q}{p}\right)^{-1}
$$

Formula (23) can be obtained by combining the formula (10)and (22). In the triaxial test, the pressure direction is regarded as the positive direction. The negative sign of the shear stress $q=-\left(\sigma_{1}-\sigma_{3}\right)$ in the formula (10) only represents its direction, and the numerical value is $\left(\sigma_{1}-\sigma_{3}\right)$, which is the same reason that the absolute value of $K$ is taken when calculating $\alpha$.

$\alpha_{1}=\frac{9}{2} \frac{\varepsilon_{v}}{3 \varepsilon_{1}-\varepsilon_{v}} \frac{\sigma_{1}+2 \sigma_{3}}{(M-3) \sigma_{1}+(2 M+3) \sigma_{3}}-1$

The volumetric strain $\varepsilon_{v}$, the axial strain $\varepsilon_{1}$ and the axial stress $\sigma_{1}$ can be measured by experiments; the confining pressure $\sigma_{3}$ is a known value; according to the critical state theory, $M$ is a quantity reflecting the ratio of test stress deviator and shear stress:

$$
M=\frac{6 \sin \varphi}{3-\sin \varphi \sin 3 \theta}
$$

where $\varphi$ is the internal friction angle of the soil sample, which can be measured by the test; $\theta$ is the Lode's angle(see Formula 7).

\section{Result analysis and discussion}

\subsection{EMD-based denoising analysis result}

\subsubsection{Test}

Test data were acquired from triaxial shear test data of the shell rockfill of a dam. A consolidated drained triaxial test was performed under constant confining pressure. The sample size was set to $300 \mathrm{~mm}$ (diameter) $\times 600 \mathrm{~mm}$ (height). The grading of the test materials is shown in Fig. 1.

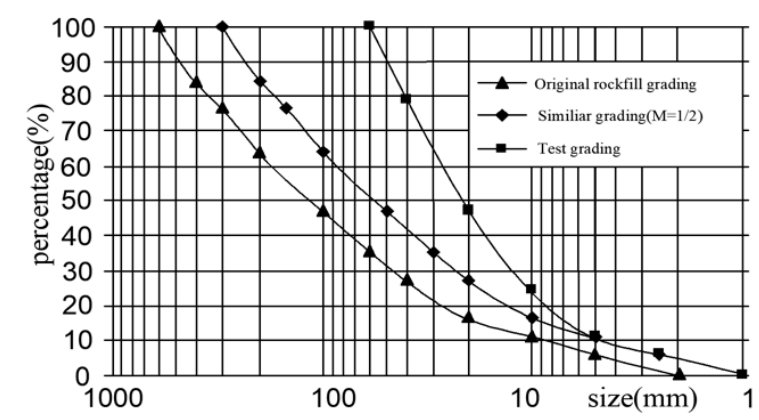

Fig. 1. Comparison of the original grading and the test grading 


\subsubsection{Denoising results}

Eighteen groups of data with an equal interval under four confining pressures $(0.5,1.3,2.2$, and $3.0 \mathrm{MPa})$ in the triaxial test were selected as the original data. The curve $d \varepsilon_{v} / d \varepsilon_{s}-q / p$ was drawn according to the original data (Fig. 2). The original data under each group of confining pressure were fitted to obtain the linear correlation equation (Figs. 3-6).

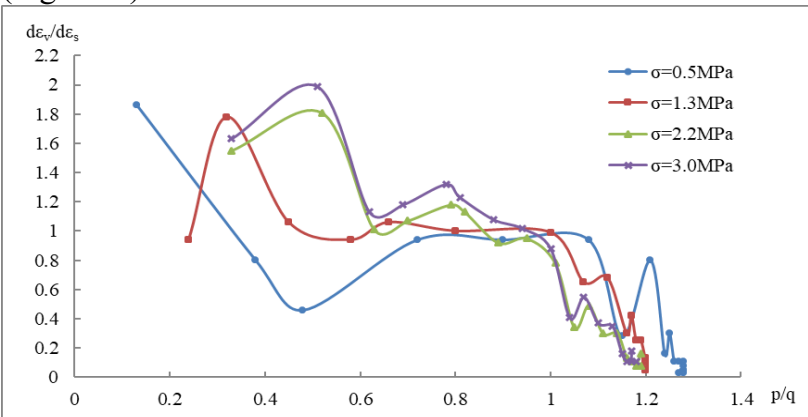

Fig.2. Statistical chart of $d \varepsilon_{v} / d \varepsilon_{s}-q / p$

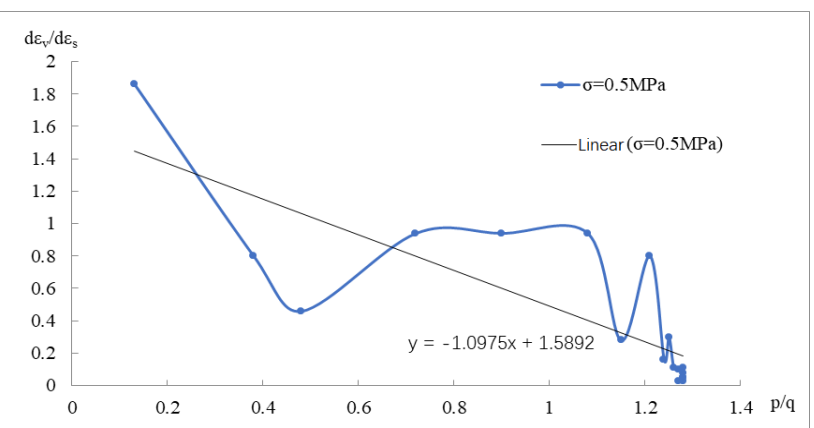

Fig.3. Fitting diagram of $\sigma=0.5 \mathrm{MPa}$

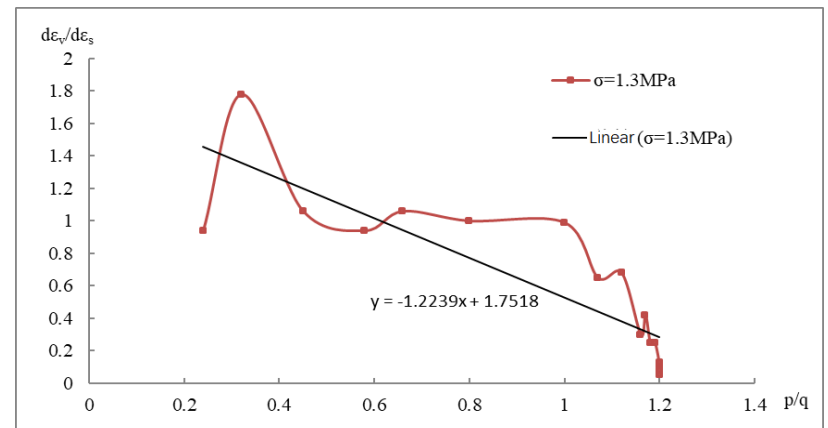

Fig.4. Fitting diagram of $\sigma=1.3 \mathrm{MPa}$

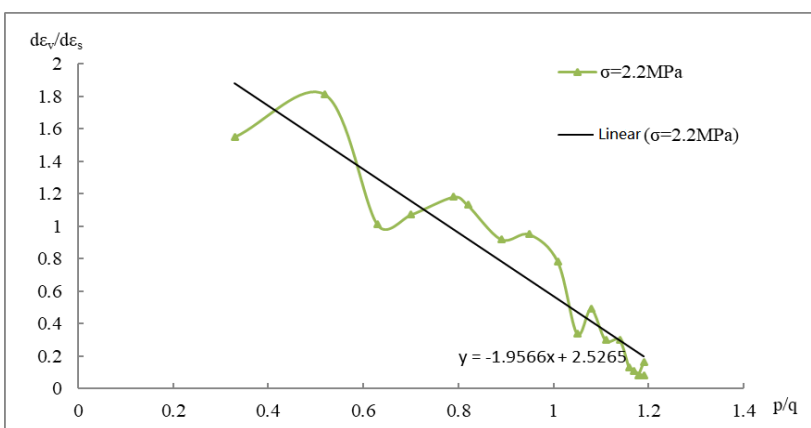

Fig. 5. Fitting diagram of $\sigma=2.2 \mathrm{MPa}$

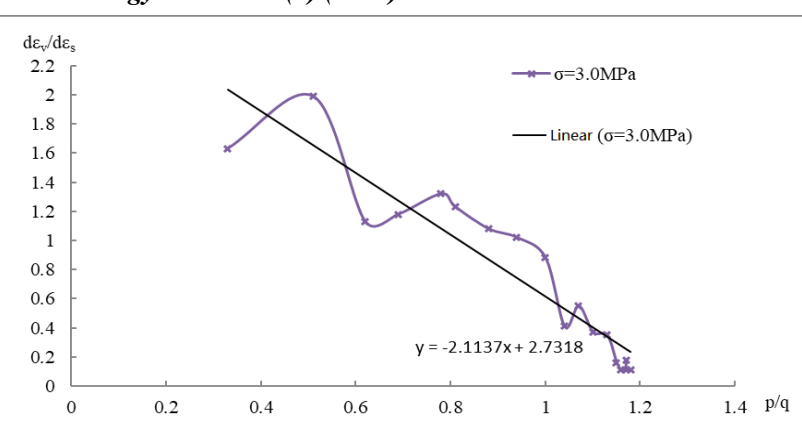

Fig. 6. Fitting diagram of $\sigma=3.0 \mathrm{MPa}$

Each group of confining data was denoised with the EMD method. The original data group $d \varepsilon_{v} / d \varepsilon_{s}-q / p$ was viewed as the time sequence of $F(x)$, and IMF function $I(x)$ and residual function $r(x)$ were derived. $I(x)$ and $r(x)$ under each confining pressure are shown in Figs. 7-10. The corresponding residual function value, which is the denoising function pertaining to $d \varepsilon_{v} / d \varepsilon_{s}-q / p$ under the corresponding confining pressure, was extracted from the drawn lines in Fig. 11.

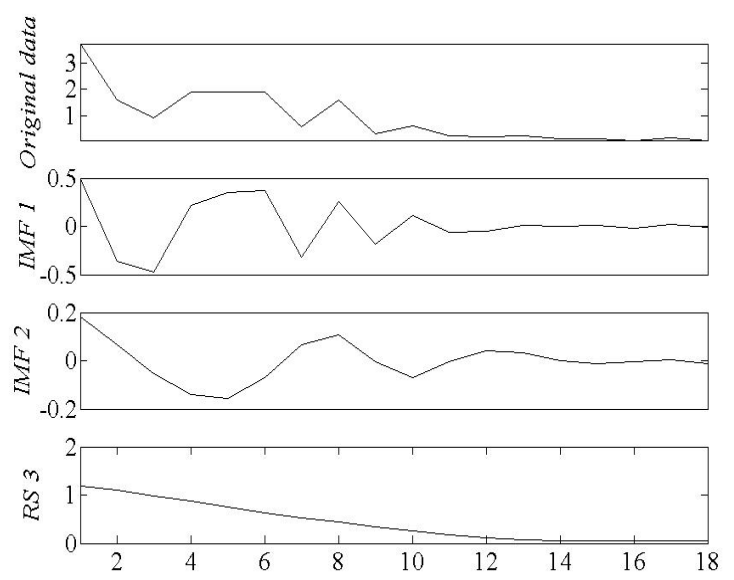

Fig.7. Denoising of $\sigma=0.5 M P a$
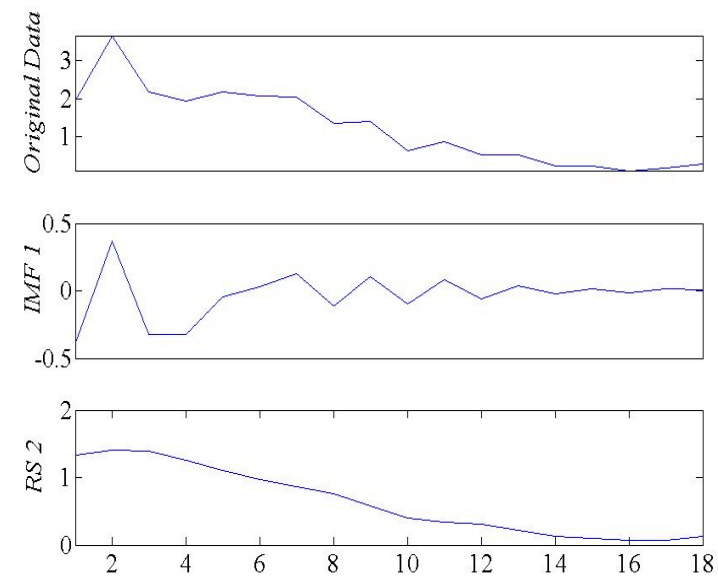

Fig.8. Denoising of $\sigma=1.3 \mathrm{MPa}$ 

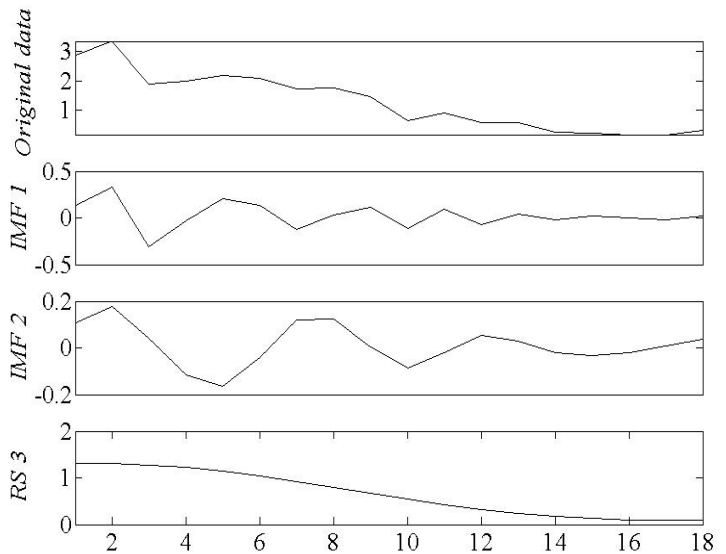

Fig.9. Denoising of $\sigma=2.2 \mathrm{MPa}$
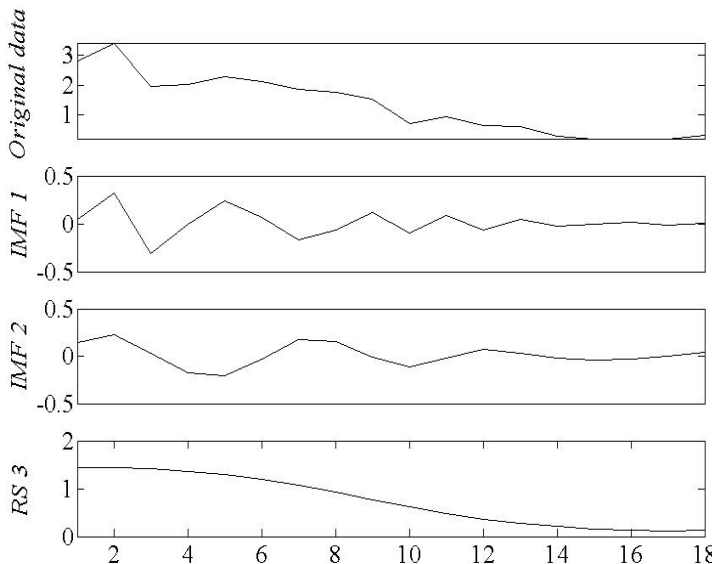

Fig.10. Denoising of $\sigma=3.0 \mathrm{MPa}$

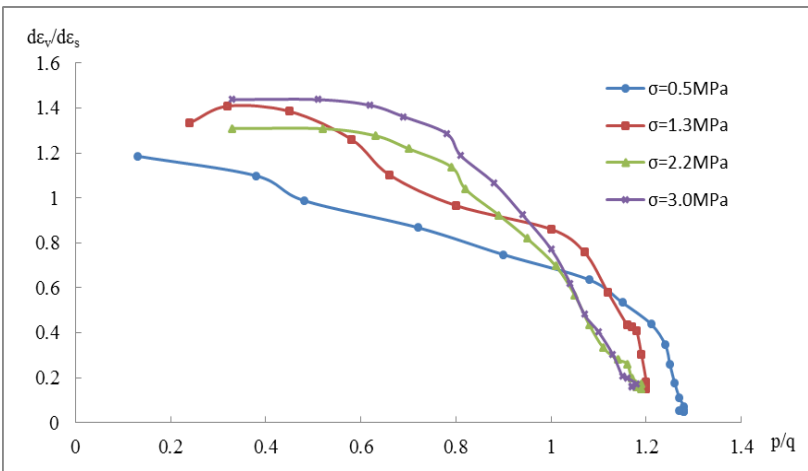

Fig. 11. Statistical data after EMD denoising

Each group of data in Fig.11 was extracted and the fitted seperately to obtain Figs.12-15. The parameters of the fitting function obtained before and after denoising are shown in Table 1 .

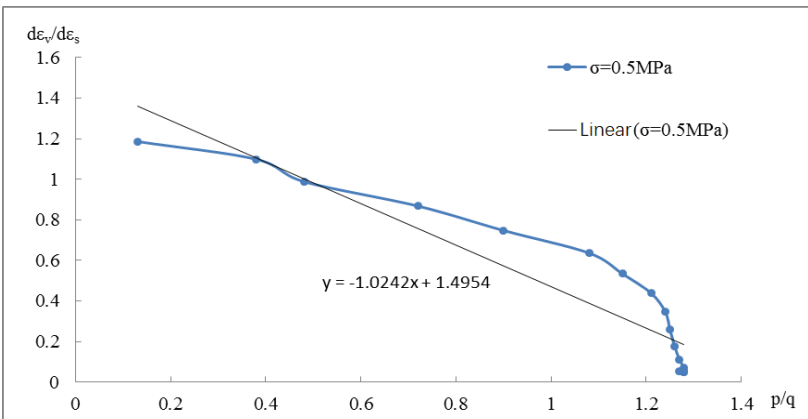

Fig.12. Data fitting after denoising of $\sigma=0.5 \mathrm{MPa}$

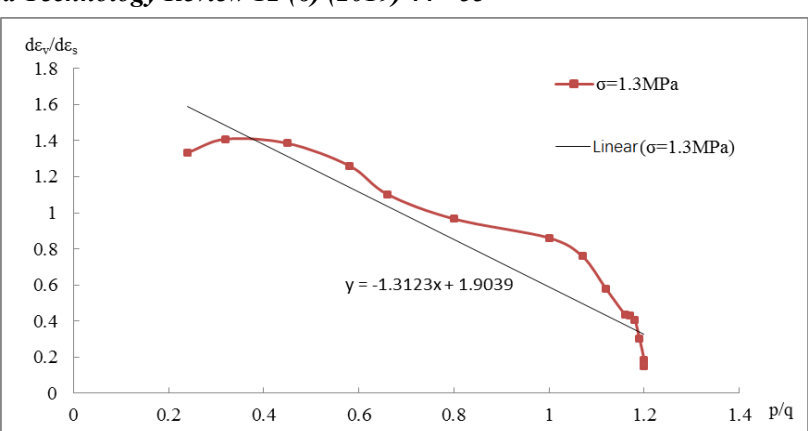

Fig. 13. Data fitting after denoising of $\sigma=1.3 \mathrm{MPa}$

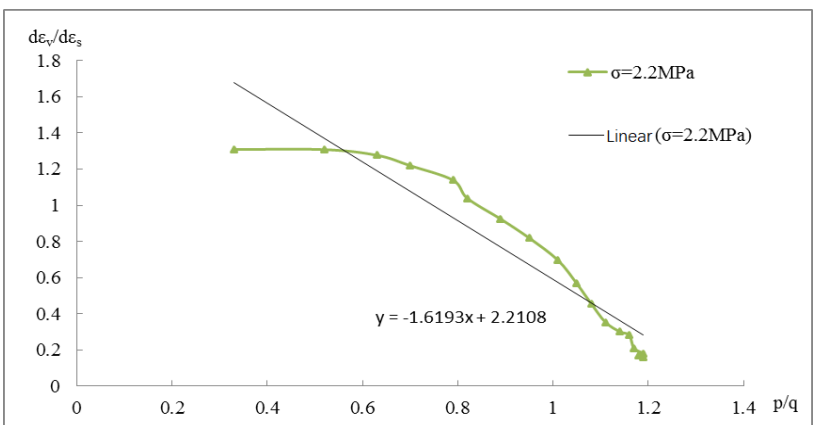

Fig. 14. Data fitting after denoising of $\sigma=2.2 \mathrm{MPa}$

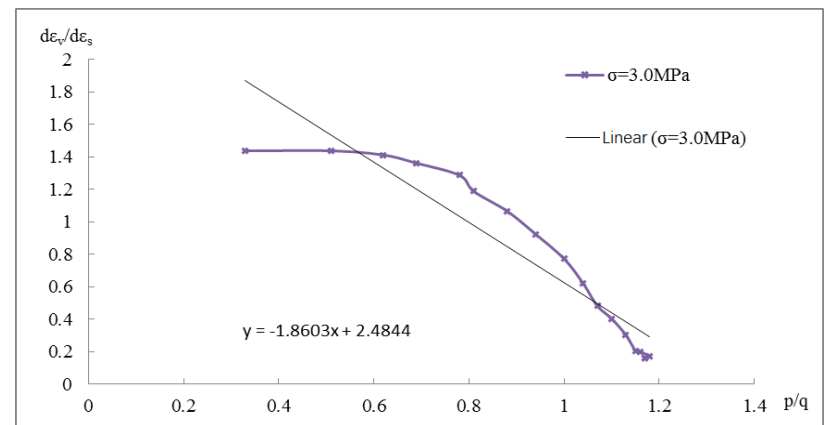

Fig. 15. Data fitting after denoising of $\sigma=3.0 \mathrm{MPa}$

Table 1. Comparison of slope before and after EMD decomposition and denoising

\begin{tabular}{c|c|c|c|c}
\hline $\begin{array}{c}\text { Parameter } \\
K\end{array}$ & $\sigma=0.5 \mathrm{MPa}$ & $\sigma=1.3 \mathrm{MPa}$ & $\sigma=2.2 \mathrm{MPa}$ & $\sigma=3.0 \mathrm{MPa}$ \\
\hline $\begin{array}{c}\text { Before } \\
\text { denoising } \\
\text { After } \\
\text { denoising }\end{array}$ & 1.0975 & 1.2239 & 1.9566 & 2.1137 \\
\hline
\end{tabular}

$\alpha=K-1$ can be derived from Formula (18), so $\alpha$ can be obtained consequently and listed in Table 2.

Table 2. Comparison of parameters $\alpha$ before and after denoising

\begin{tabular}{c|c|c|c|c}
\hline $\begin{array}{c}\text { Parameter } \\
\alpha\end{array}$ & $\sigma=0.5 M P a$ & $\sigma=1.3 M P a$ & $\sigma=2.2 M P a$ & $\sigma=3.0 M P a$ \\
\hline $\begin{array}{c}\text { Before } \\
\text { denoising } \\
\begin{array}{c}\text { After } \\
\text { denoising }\end{array}\end{array}$ & 0.0975 & 0.2239 & 0.9566 & 1.1137 \\
\hline
\end{tabular}

Positive or negative values of parameter $K$ from the fitting function represent an upward or downward trend of image data. In this study, parameter $\alpha$ was unrelated with positive and negative values of $K$. Therefore, the absolute value of $K$ was selected in statistics of $\alpha$. Table 2 shows 
the numerical values of parameter $\alpha$. The confining pressures in the test before and after denoising were compared. When the confining pressure increased, parameter $\alpha$ increased gradually and fluctuated within a certain range. This phenomenon conforms to the hypothesis of critical state theory. Parameter $\alpha$ is a material property, and it implies the Poisson's ratio. Confining pressure can influence parameter $\alpha$ significantly.

\subsection{Optimization of parameter $\alpha$ based on cloud theory}

The parameter sequences before and after denoising were transformed into digital features $\left[x_{1}\left(E x_{1}, E n_{1}, H e_{1}\right)\right.$ and $x_{2}\left(E x_{2}, E n_{2}, H e_{2}\right)$ in Table 3] required by cloud theory by using a forward cloud generator. A total of 1000 forward cloud droplets were generated for both digital features by using the forward cloud generator (Figs. 16 and 17). The cloud droplet cluster indicated that parameter $\alpha$ was improved from the conceptual layer to the numerical layer, which is the first step of cloud theory: transformation from "qualitative to quantitative." In accordance with the " $3 E_{n}$ criteria" of cloud theory [32], the value ranges of parameter $\alpha$ were determined to be $\alpha_{1} \in(0,2.24)$ and $\alpha_{2} \in(0,1.5283)$. The value ranges were defined as the range in which the calculated parameter $\alpha$ has $99.7 \%$ of probability falls after several tests. Numerical values of cloud droplets beyond these ranges can be ignored. Determining the value range of parameter $\alpha$ is the second step of cloud theory: transformation from "quantitative to qualitative." After completing cloud theory, 1000 values of parameter $\alpha$ that use the four parameters as the internal law were generated from four $\alpha$ parameters. Moreover, the value ranges of each $\alpha$ were determined.

Table. 3. Cloud theory parameter calculation

\begin{tabular}{c|c|c|c|l|c|c}
\hline \multirow{2}{*}{ Index } & \multicolumn{2}{|c|}{$E x$} & \multicolumn{2}{c|}{$E n$} & \multicolumn{2}{c}{$H e$} \\
\cline { 2 - 7 } & $E x_{1}$ & $E x_{2}$ & $E n_{1}$ & $E n_{2}$ & $H e_{1}$ & $H e_{2}$ \\
\hline Parameter & 0.5979 & 0.4540 & 0.5478 & 0.0833 & 0.1961 & 0.0647 \\
\cline { 1 - 5 } $\begin{array}{c}\text { Relative } \\
\text { error }\end{array}$ & \multicolumn{2}{|c|}{$24 \%$} & \multicolumn{2}{c|}{$35 \%$} & \multicolumn{2}{c}{$67 \%$} \\
\hline
\end{tabular}

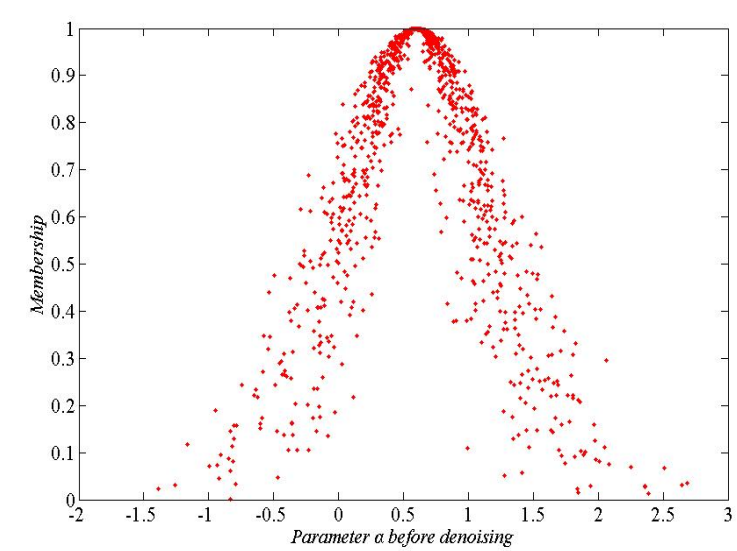

Fig.16. Cloud droplet of $\alpha$ before denoising

(1) Entropy and hyper entropy are parameters reflecting the discreteness degree of data. Small values of entropy and hyper entropy indicate a strong data correlation. Table 3 shows that the fitted formula has good effects. The entropy and hyper entropy after denoising were $35 \%$ and $67 \%$ lower than the previous results. The EMD method decreased the data error significantly;
(2) Comparison of Figs. 16 and 17 shows that the parameter $\alpha$ values after denoising were more concentrated toward what is expected than the previous values, thus decreasing the fluctuation range of the parameters. In this study, the " $3 E n$ principle" of cloud theory proved that the value range of parameter $\alpha$ is more accurate after denoising with the EMD method. The cloud droplet range of parameter $\alpha$ after denoising is $32 \%$ narrower than that before.

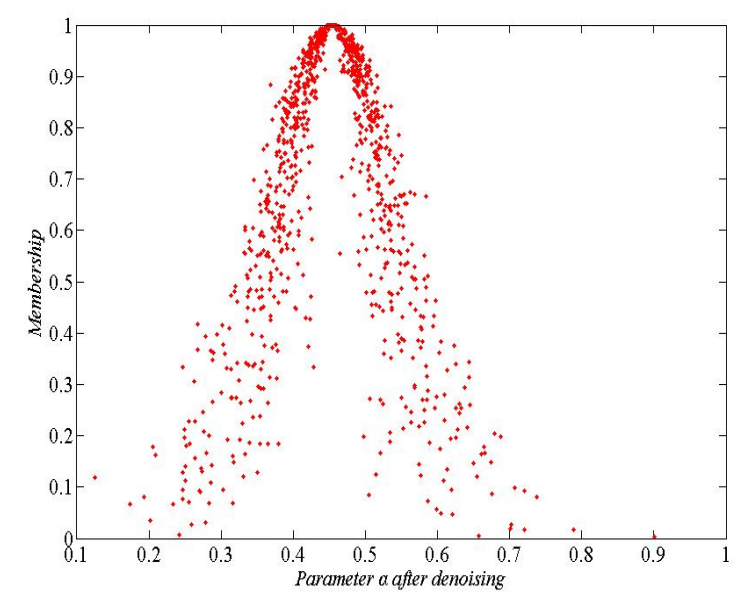

Fig.17. Cloud droplet of $\alpha$ after denoising

\subsection{Optimization result analysis of the calculation formula}

A modification parameter $k=\frac{\alpha_{2}}{\alpha_{1}}$ was introduced.

According to the calculation results of cloud theory, cloud droplets before and after denoising were matched using the modification formulaas the calculation principle, thereby generating new cloud droplet cluster $k$ (Fig. 18). The cloud map implied that the characteristic numerical values of $k$ are $k\{0.75,0.13,0.027\}$. Then, the value range of $k \in(0.37,1.14)$ was derived according to the " $3 E n$ principle" of cloud theory.

Taking the correction coefficient $k$ into formula (23), we obtain the modified formula:

$$
\begin{aligned}
& \alpha=k \alpha_{1} \\
& =\frac{9 k}{2} \frac{\varepsilon_{v}}{3 \varepsilon_{1}-\varepsilon_{v}} \frac{\sigma_{1}+2 \sigma_{3}}{(M-3) \sigma_{1}+(2 M+3) \sigma_{3}}-k \\
& k \in(0.37,1.14)
\end{aligned}
$$

where $\frac{9 k}{2}$ is the a correction parameter; $\frac{\varepsilon_{v}}{3 \varepsilon_{1}-\varepsilon_{v}}$ reflects the shape change of the soil itself; $\frac{\sigma_{1}+2 \sigma_{3}}{(M-3) \sigma_{1}+(2 M+3) \sigma_{3}}$ reflects the external force of the soil; $k$ is a correction parameter.

\subsection{Simulation results analysis of the $\mathrm{P}-\mathrm{Z}$ model}

With formula (25), $\alpha_{1}=0.60 ; \alpha_{2}=0.45$ were calculated. The constant parameters of the P-Z model, $\alpha_{1}=0.60$ and $\alpha_{2}=0.45$, were determined for numerical simulation analysis of triaxial test data. The calculated results and 
experimental data points were compared. The results are shown in Figs. 19-22.

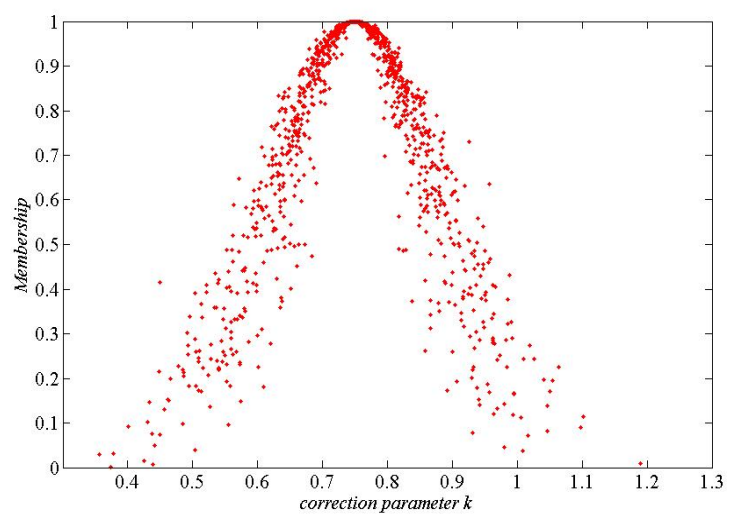

Fig.18. Correction parameter $k$ cloud droplet

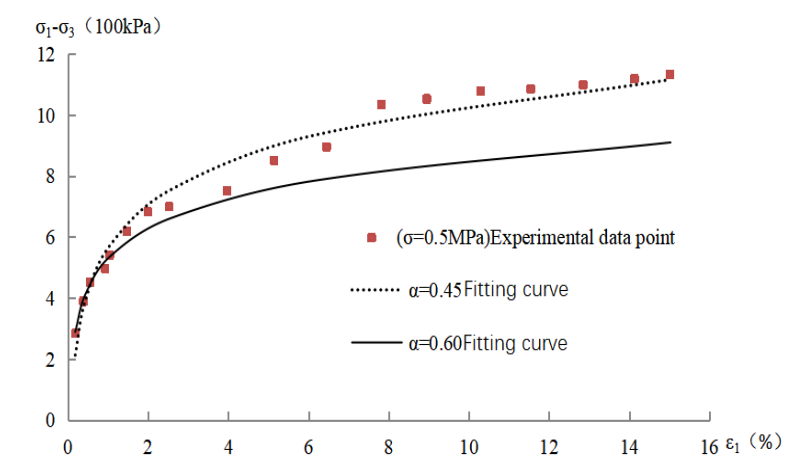

Fig.19. Comparison Fitting diagram of $\sigma=0.5 \mathrm{MPa}$

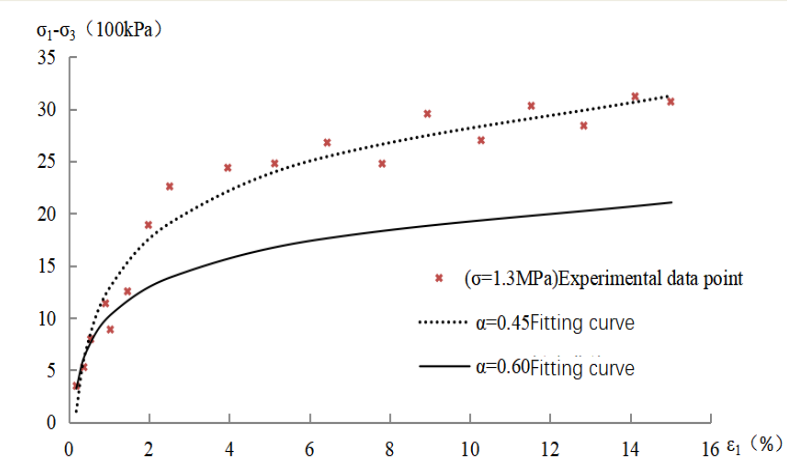

Fig.20. Comparison Fitting diagram of $\sigma=1.3 \mathrm{MPa}$

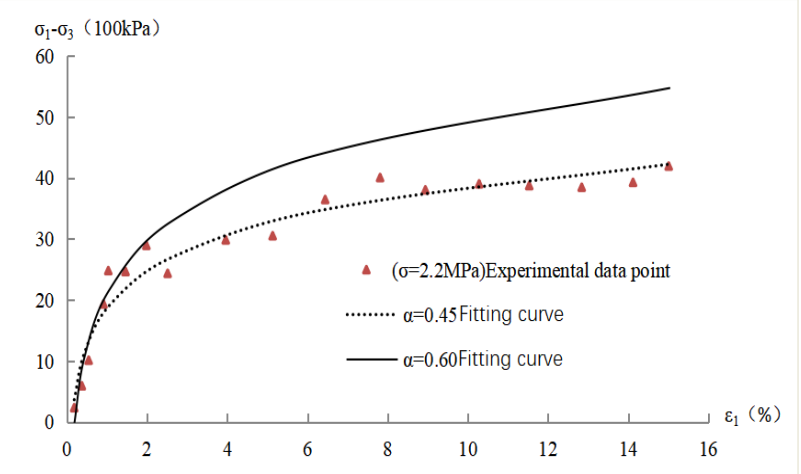

Fig.21. Comparison Fitting diagram of $\sigma=2.2 \mathrm{MPa}$

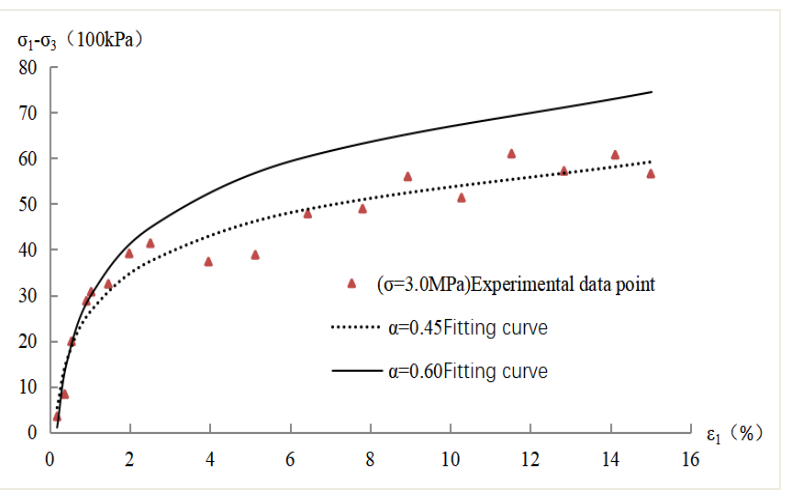

Fig.22. Comparison Fitting diagram of $\sigma=3.0 \mathrm{MPa}$

In Figs. 19-22, the solid lines are the simulation results at $\alpha_{1}=0.60$, and the dotted lines are the simulation results at $\alpha_{2}=0.45$. Comparison of these figures indicated that the results at $\alpha_{2}=0.45$ presented the best fitting effect with experimental scattering points, and they approached the test data. However, the results at $\alpha_{1}=0.60$ deviated from the experimental scattering points. The results at $\alpha_{1}=0.60$ were above and below the test data and showed large errors. Therefore, the parameter $\alpha$ optimized by EMD denoising and cloud theory can increase calculation accuracy.

\section{Conclusions}

The parameter $\alpha$ of the P-Z model was optimized through the EMD method and cloud theory based on triaxial shear test data of earth-rockfill dam materials. The optimized parameters were applied to the simulation analysis. The following major conclusions were obtained.

(1) Triaxial shear test data were denoised by the EMD method and compared with the original data. The denoised data presented more irregular change laws, and the correlation of sequences was stronger than that of original data;

(2) Parameter $\alpha$ of the P-Z model was optimized by cloud theory. The results showed that the accuracy of the value range of the optimized parameter $\alpha$ increased by $32 \%$, and the discreteness was reduced significantly;

(3) The P-Z model after the optimization of parameter $\alpha$ was used for a simulation analysis of the triaxial shear test data. The calculated and test results were consistent, indicating that the optimized P-Z model can simulate the mechanical properties of earth-rockfill dam materials effectively.

In summary, the parameter $\alpha$ of the P-Z constitutive model was optimized with the EMD method and cloud theory, which has a clear concept, a reasonable method, reliable results, and low discreteness. This work lays foundations for accurate simulations of the mechanical properties of earth-rockfill dam materials by using the $\mathrm{P}-\mathrm{Z}$ model. However, various types of earth-rockfill dam materials are available, and their mechanical properties differ significantly. This study focused only on coarse particle materials. Future studies can optimize the parameters of the P-Z model for clay and sandy soils to expand the application range of the model. Such research would help understand the mechanical properties of earthrockfill dam materials under numerous complicated stress states. 


\section{Zhang Hongyang, Zhang Wenxin, Ding Zelin, Li Shuai and Ge Wei/ \\ Journal of Engineering Science and Technology Review 12 (6) (2019) 44 - 53}

\section{Acknowledgements}

The authors are grateful for the support provided by the National Natural Science Foundation of China(51609087, 51709114), National Key Research and Development Plan Project(2017YFC1501201), Key project of scientific research in Colleges and Universities of Henan Province (17A570004), Collaborative Innovation Center of Water Resources Efficient Utilization and Protection Engineering,
Henan Province, and 2017 Famous Teacher Training Program of NCWU.

This is an Open Access article distributed under the terms of the Creative Commons Attribution License

\section{References}

1. Zhang, H. Y., "Stability evaluation and engineering application of earth-rock dam in strong earthquake area". Beijing: Water and Power Press, China, 2014, pp.1-20.

2. Duncan, J. M., Chang, C. Y., "Nonlinear analysis of stress and strain in soils". Journal of Soil Mechanics \& Foundations Division, 96(05), 1970, pp.1629-1653.

3. Sun, M. Q., Liu, Y., He, M., "Adaptive research of nonlinear K-G model to CSG material". Yellow River, 35(07), 2013, pp.96$98+101$.

4. Drucker, D. C., Prager, W., "Soil mechanics and plastic analysis or limit design". Quarterly of Applied Mathematics, 10(02), 1952, pp.157-165.

5. Schofield, A., Wroth, P., "Critical state soil mechanics". London: McGraw-Hill, United Kingdom, 1968, pp.55-70.

6. Shen, Z. J., "Mathematical model of soil structure-the core problem of soil mechanics in the 21st Century". Chinese Juornal of Geoteehnieal Engineering, 18(01), 1996, pp.95-97.

7. Pastor, M., Zienkiewicz, O. C., Leung, K. H., "Simple model for transient soil loading in earthquake analysis. II. Non-associative models for sands". International Journal for Numerical and Analytical Methods in Geomechanics, 9(05), 1985, pp.477-498.

8. Zienkiewicz, O. C., Mroz, Z., "Generalized plasticity formulation and applications to geomechanics". Mechanics of Engineering Materials, 44(03), 1984, pp.655-680.

9. Mroz, Z., Zienkiewicz, O. C., "Uniform formulation of constitutive equations for clays and sands". Mechanics of Engineering Materials, 12, 1984, pp.415-449.

10. Mroz, Z., Dieter, W., Stanislaw, D., "Inelastic behaviour of structures under variable loads". Berlin: Springer Science \& Business Media, Germany, 2012, pp.157-169.

11. Pastor, M., Chan, A. H. C., Mira, P., et al. "Computational geomechanics: the heritage of Olek Zienkiewicz". International Journal for Numerical Methods in Engineering, 87(1-5), 2011, pp.457-489.

12. Jefferies, M., Been, K., "Soil liquefaction: a critical state approach". Boca Raton: CRC Press, United States, 2015, pp.95104.

13. Zhang, H. Y., Han, L, W., Zhang, X. Q., et al. "The P-Z model for core materials of earth-rockfill dam". International Journal of Earth Sciences and Engineering, 7(02), 2014, pp.668-774.

14. Zhang, H. Y., Li, S., Zhang, X. Q., et al. "Research on method of dynamic stability analysis for slopes of earth and rockfill dam basing on the P-Z model'. Technical Gazette, 25(01), 2018, pp.230235.

15. Chen, S. S., Peng, C., Fu, Z. Z., "Dynamic constitutive model for rockfill materials based on generalized plasticity theory". Chinese Journal of Geotechnical Engineering, 34(11), 2012, pp.1961-1968.

16. Gröchenig, K., "Foundations of time-frequency analysis". Berlin: Springer Science \& Business Media, Germany, 2013, pp.33-42.

17. Zhang, J. W., Bao, Z. L., Jiang, Q., et al. "Research on characteristics information identification for flood discharge structure based on SVD and improved EMD". Journal of Basic Science and Engineering, 24(04), 2016, pp. 698-711.
18. Li, C. Y., Lian, J. J., Liu, F., et al. "An improved filtering method based on EMD and wavelet-threshold and its application in vibration analysis for a flood discharge structure". Journal of Vibration and Shock, 32(19), 2013, pp.63-70+110.

19. Su, H. Z., Yang, M., Wen, Z. Q., et al. "Deformation-based safety monitoring model for high slope in hydropower project". Journal of Civil Structural Health Monitoring, 6(05), 2016, pp.779-790.

20. Li, D., "Membership clouds and membership cloud generators". Journal of Computer Research and Development, 32(06), 1995, pp.15-20.

21. Wang, G., Xu, C. L., Li, D. Y., "Generic normal cloud model". Information Sciences, 280(280), 2014, pp.1-15.

22. Liu, Y. C., Li, D. Y., He, W., et al. "Granular computing based on gaussian cloud transformation". Fundamenta Informaticae, 127(14), 2013, pp.385-398.

23. Wang, J. Q., Peng, J. J., Zhang, H. Y., et al. "An uncertain linguistic multi-criteria group decision-making method based on a cloud model". Group Decision \& Negotiation, 24(01), 2015, pp.171-192.

24. Tan, Z. Y., "Discussion on dam safety evaluation cloud model application". Water Conservancy Construction and Management, 36(09), 2016, pp.52-55.

25. Zhang, T., "Dam safety evaluation based on normal cloud model and its application". Water Power, 43(05), 2017, pp.112-118.

26. Wang, F., Zhong, D. H., Yan, Y. L., et al. "Rockfill dam compaction quality evaluation based on cloud-fuzzy model". Journal of Zhejiang University-Science A, 19(04), 2018, pp.289303.

27. Xiang, D., Ge, S., "Method of fault feature extraction based on EMD sample entropy and LLTSA". Journal of Aerospace Power, 29(07), 2014, pp.1535-1542.

28. Liu, J., Wen, G. L., "Continuum topology optimization considering uncertainties in load locations based on the cloud model". Engineering Optimization, 50(06), 2017, pp.1041-1060.

29. Peng, H. G., Wang, J. Q., "A multicriteria group decision-making method based on the normal cloud model with Zadeh'sz-numbers". Institute of Electrical and Electronics Engineers Transactions on Fuzzy Systems, 26(06), 2018, pp.3246-3260.

30. Wang, D., Liu, D., Ding, H., et al. "A cloud model-based approach for water quality assessment". Environmental Research, 148, 2016, pp.24-35.

31. Liu, Y., Li, D. Y., "Statistics on atomized feature of normal cloud model". Journal of Beijing University of Aeronautics and Astronautics, 36(11), 2010, pp.1320-1324.

32. Wang, X. T., Li, S. C., Xu, Z. H., et al. "Risk assessment of water inrush in karst tunnels excavation based on normal cloud model". Bulletin of Engineering Geology and the Environment, 78(05), 2019, pp.3783-3798. 